UDC 547.32

\title{
HALOALKOXYLATION OF 3-ORGANYLOXY-1-PROPENES IN DIMETHYLACETYLENECARBINOL MEDIUM
}

\author{
A.R. Ezizbeyli, G.M. Talybov, E.H. Mammadbayli \\ Y.H. Mammadaliyev Institute of Petrochemical Processes \\ Naional Academy of Sciences of Azerbaijan \\ Khojaly ave. 30, AZ 1025, Baku, Azerbaijan \\ e-mail:ezizbeyli83@mail.ru,ahmed_adna@rambler.ru,eldar_mamedbeyli@mail.ru
}

Received 08.11.2019

\begin{abstract}
Reaction of alkoxyhalogenation 3-organyloxy-1-propenes with N-bromosuccinimide (or iodine) in dimethylacetylenecarbinol medium proceeds regioselectively according to NMR spectra. It proceeds at a low temperatureç cooling the reaction mixture with ordinary ice. The main reaction products, diethers - 3-bromo (iodine) -1,2-propanediol, are formed in high yields. It founded that the alkoxyhalogenation of allyl ethers with propinol proceeds regioselectively with fixation of the halogen atom to the peripheral carbon atom of $C=C$ bond. The yields of bromine-containing products are higher than those for iodine analogues. Iodine-containing products boil at a higher temperature than bromine analogues. In the reaction products, substances with the replacement of the halogen atom with unsaturated alcohol are not observed. The reaction was monitored by means of thin layer chromatography. Similar synthesized compounds are used as biologically active compounds.

Keywords: alkoxyhalogenation, $C_{3}$-unsaturated alcohols, $\beta$-halogenethers, FT-IR spectra, ${ }^{l} H$ NMR spectra
\end{abstract}

DOI: $10.32737 / 2221-8688-2020-1-102-105$

\section{Introduction}

Alkoxyhalogenation of alkenes [1,2] with $\mathrm{C}_{3}$-unsaturated alcohols is a common method for the synthesis of unsaturated $\beta$ - halogenethers which are used in the synthesis of heterocyclic compounds [4-6] and biologically active preparations [3-9].

\section{Experimental}

The reaction was carried out at low same process in the temperature range $(-5 \div 10$ temperature $\left(-5 \div 0{ }^{\circ} \mathrm{C}\right)$ in order to prevent $\left.{ }^{\circ} \mathrm{C}\right)$ was observed yield reduction (I-VI) by tarring process. Upon implementation the about half.

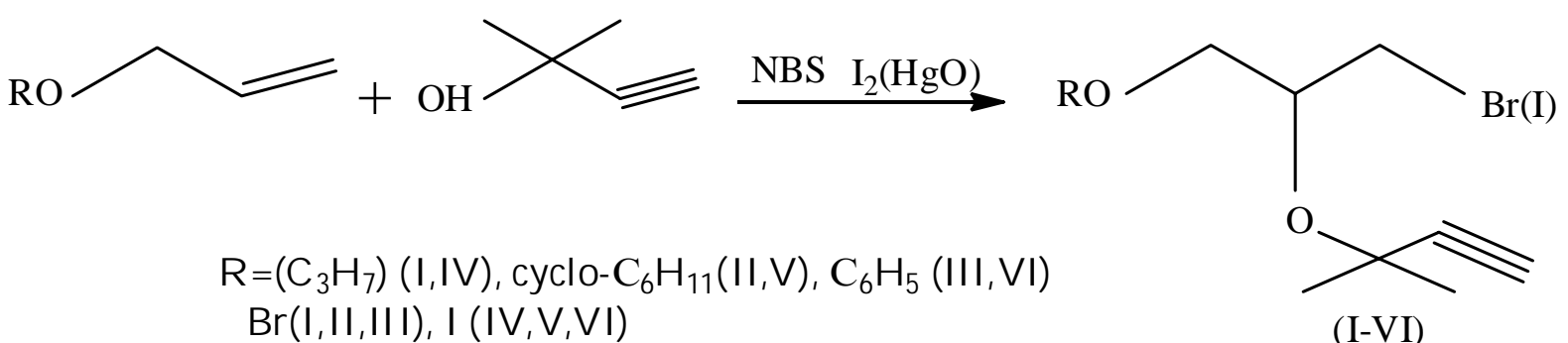

We found that the alkoxyhalogenation of allyl ethers with propinol proceeds regioselectively with fixation of the halogen atom to the peripheral carbon atom of $\mathrm{C}=\mathrm{C}$ bond. Regioselectivity of reactions (according to the Markovnikov rule) is confirmed by ${ }^{1} \mathrm{H}$ NMR spectral data of compounds (I-VI) which says that the presence of doublet signals of protons in methylene groups bonded to a halogen atom due to spin-spin interaction with 
methine protons in the region of $3.28 \mathrm{dd}(1 \mathrm{H}, \mathrm{J}$ $\left.=10.3,5.8 \mathrm{~Hz} \mathrm{CH} \mathrm{CH}_{2} \mathrm{I}\right), 3.41 \mathrm{dd} .(1 \mathrm{H}, \mathrm{J}=10.3$, $\left.5.8 \mathrm{~Hz} \mathrm{CH}_{2} \mathrm{I}\right)$ for $\mathrm{CH}_{2} \mathrm{I}$ and $3.38 \mathrm{dd}(1 \mathrm{H}$, $\left.\mathrm{J}=10.5,5.7 \mathrm{~Hz} \mathrm{CH}_{2} \mathrm{Br}\right)$ and $3.42 \mathrm{dd} .(1 \mathrm{H}$, $\mathrm{J}=10.5,5.7 \mathrm{~Hz} \mathrm{CH} \mathrm{CH}_{2} \mathrm{I}$, for $\mathrm{CH}_{2} \mathrm{Br}$ respectively.

In the FT-IR spectra of synthesized compounds (I-VI), absorption bands are observed in the region of $1150-1085 \mathrm{~cm}^{-1}$ due to asymmetric stretching vibrations of the $\mathrm{C}$ $\mathrm{O}-\mathrm{C}$ bond. The bands in the regions of 3333$3267 \mathrm{~cm}^{-1}$ and 2140-2100 $\mathrm{cm}^{-1}$ are characteristic for stretching vibrations of monosubstituted acetylenes.

The synthesized compounds are readily soluble in organic solvents and stable when stored under normal conditions.

This method allows to synthesize 3-halo1,2-propanediol diethers in $60-70 \%$ yield.

The composition and structure of the obtained compounds are established on the basis of FTIR and ${ }^{1} \mathrm{H}$ NMR spectral data.

\section{Results and discussion}

FT-IR spectra of compounds were measured on Specord-75 instruments in the microlayer and using $\mathrm{KBr}$ disks. ${ }^{1} \mathrm{H}$ NMR spectra of substances in the solution $\mathrm{CDCl}_{3}$ recorded on «Bruker SF-400" (300.134 MHz) device, an internal standard HMDS.

\section{3-[(1-Bromo-3-propyloxypropan-2-} yl)oxy]-3-methylbut-1-in (I). It was gradually added with $44 \mathrm{~g}$ of N-bromosuccinimide (NBS) to a cooled equimolar mixture of $25 \mathrm{~g}$ allylpropyl ether and $14 \mathrm{~g}$ propargyl alcohol with stirring, so that a temperature of the reaction mixture could not exceed $0{ }^{\circ} \mathrm{C}$. The stirring was carried out for a period 5 hours at a room temperature. The succinimide formed was filtered off, and the mixture was alkalized by solution of $15 \mathrm{~g}$ of sodium hydroxide in $100 \mathrm{ml}$ of water, then extracted with ether and dried with $\mathrm{CaCl}_{2}$. Following ether removal, the resudio was distilled by means of vacuum distillation method. Allocated $41.15 \mathrm{~g}(70 \%)$ of compound (I) T. boil. $65-66{ }^{\circ} \mathrm{C}(2 \mathrm{mmHg}$ art.) $d_{4}^{20} 1.2728, \quad \eta_{20}^{D} 1.4924 . \quad \mathrm{MR}_{\mathrm{D}}$ 53.63, calcd. 52,95. FT-IR spectra $\left(\mathrm{v} / \mathrm{sm}^{-1}\right): 620(\mathrm{C}-$ $\mathrm{Br}), 1100(\mathrm{C}-\mathrm{O}-\mathrm{C}), 2100$ and $3300(\mathrm{C} \equiv \mathrm{CH})$. ${ }^{1} \mathrm{H}$ NMR $\left(\delta, p p m ., \mathrm{CDCl}_{3}\right): 0.75 \mathrm{t}\left(3 \mathrm{H}, \mathrm{CH}_{3}\right.$, $\mathrm{J}=7.5 \mathrm{~Hz}), 1.08 \mathrm{~s}\left(6 \mathrm{H}, 2 \mathrm{CH}_{3}\right) 1.21 \mathrm{k}\left(2 \mathrm{H}, \mathrm{CH}_{2}\right.$, $\mathrm{J}=7.5 \mathrm{~Hz}), 2.48 \mathrm{~s}\left({ }^{1} \mathrm{H}, \mathrm{CH}\right), 3.21 \mathrm{~m}(4 \mathrm{H}$, $\left.\mathrm{CH}_{2} \mathrm{OCH}_{2}\right), 3.38 \mathrm{dd}\left({ }^{1} \mathrm{H}, \mathrm{CH}_{2} \mathrm{Br}, \mathrm{J}=10.5,5.7\right.$ $\mathrm{Hz}), 3.42 \mathrm{dd}\left({ }^{1} \mathrm{H}, \mathrm{CH}_{2} \mathrm{Br}, \mathrm{J}=10.5,5.7 \mathrm{~Hz}\right), 3.85$ $\mathrm{m}\left({ }^{1} \mathrm{H}, \mathrm{CH}\right)$. Found \%: C 50.22, H 7.30, Br 30.38. $\mathrm{C}_{11} \mathrm{H}_{19} \mathrm{BrO}_{2}$. Calculated,\%: C 50.20, H 7.28, Br 30.36 .

Similarly based on:

-allylcyclohexyl ether, propargyl alcohol and NBS was obtained 3-Bromo-2-[(2metylbut-3-in-2-yl)oxy]propyl cyclohexane (II) by yield of $65 \%$, T.boil. $80-82^{\circ} \mathrm{C}(2 \mathrm{~mm}$
$\mathrm{Hg}$ art. $), d_{4}^{20} 1.2763, \eta_{20}^{D}$ 1.5060. $\mathrm{MR}_{\mathrm{D}}$ 64.06, calcd. 64.68. FT-IR spectra $\left(v / \mathrm{sm}^{-1}\right): 628(\mathrm{C}-$ $\mathrm{Br}), 1140(\mathrm{C}-\mathrm{O}-\mathrm{C}), 2100$ and $3300(\mathrm{C} \equiv \mathrm{CH})$. ${ }^{1} \mathrm{H}$ NMR ( $\delta$, ppm., $\left.\mathrm{CDCl}_{3}\right): 0.782-1.24 \mathrm{~m}$, $\left(11 \mathrm{H}, 1.09 \mathrm{~s},\left(6 \mathrm{H}, 2 \mathrm{CH}_{3}\right)\right.$ protons of the cycle $)$, $2.48 \mathrm{~s}(1 \mathrm{H} \equiv \mathrm{CH}), 3.39 \mathrm{dd}\left(1 \mathrm{H}, \mathrm{CH}_{2} \mathrm{Br}, \mathrm{J}=10.5\right.$, $5.7 \mathrm{~Hz}), 3.42 \mathrm{dd}\left(1 \mathrm{H}, \mathrm{CH}_{2} \mathrm{Br}, \mathrm{J}=10.5,5.7 \mathrm{~Hz}\right)$, $3.83 \mathrm{~m}(1 \mathrm{H}, \mathrm{CH})$. Found \%: C 55.47, H 7.67, Br 26.37. $\mathrm{C}_{14} \mathrm{H}_{23} \mathrm{BrO}_{2}$. HMDS. calculated, $\%$ : C 55.45, H 7.65, Br 26.35.

- allylphenyl ether, propargyl alcohol and NBS 3-Bromo-2-[(2-methylbut-3-yn-2yl)oxy]propyl \}benzene (III) obtained by yield of $68.5 \%$, T.boil. $99-100{ }^{\circ} \mathrm{C} d_{4}^{20} 1.2894, \eta_{20}^{D}$ 1.5256. $\mathrm{MR}_{\mathrm{D}}$ 64.04,. calculated,\%: 63.52 . Found \%: C 56.60, H 5.79, Br 26.91. $\mathrm{C}_{14} \mathrm{H}_{17} \mathrm{BrO}_{2}$. HMDS. calculated,\%: C 56.58, H 5.77, $\mathrm{Br} 26.89$.

3 - [(1-Iodo-3-propoxypropan-2-yl) oxy] 3-methylbut-1-in (IV). It was added $26 \mathrm{~g}$ $(0.12 \mathrm{~mol})$ of $\mathrm{HgO}$ to a cooled $\left(-5 \div 0{ }^{\circ} \mathrm{C}\right)$ and intensively stirred mixture of $14 \mathrm{~g}(0.25 \mathrm{~mol})$ of propargyl alcohol and $25 \mathrm{~g}(0.25 \mathrm{~mol})$ of allyl propyl ether ,then $63.45 \mathrm{~g}(0.25 \mathrm{~mol})$ of finely crushed crystal of iodine ( $1 \mathrm{~g}$ each). The stirring was carried out for a period 3-4 hours at a room temperature, the mixture was filtered, the filtrate washed by $\mathrm{Na}_{2} \mathrm{~S}_{2} \mathrm{O}_{3}$ solution and extracted with ether. The extract is dried using $\mathrm{CaCl}_{2}$. Ether was removed in a rotary evaporator, and the residue was recrystallized. Received $47.9 \mathrm{~g}(68 \%)$ of substance (IV), melt.point 52-53 ${ }^{\circ} \mathrm{C}$ (from heptane). FT-IR spectra $\left(v / \mathrm{sm}^{-1}\right): 550(\mathrm{C}-\mathrm{I})$, $1100(\mathrm{C}-\mathrm{O}-\mathrm{C}) 2100$ and $3300(\mathrm{C} \equiv \mathrm{CH}) .{ }^{1} \mathrm{H}$ NMR $\left(\delta\right.$, ppm., $\left.\mathrm{CDCl}_{3}\right): 0.75$ t. $\left(3 \mathrm{H}, \mathrm{CH}_{3}\right)$, $1.11 \mathrm{~s}\left(6 \mathrm{H}, 2 \mathrm{CH}_{3}\right), 1.24 \mathrm{~m} .\left(2 \mathrm{H}, \mathrm{CH}_{2}\right), 2.43 \mathrm{~s}$. $(1 \mathrm{H}, \equiv \mathrm{CH}), 3.21-3.43 \mathrm{~m} .\left(4 \mathrm{H}, \mathrm{CH}_{2} \mathrm{OCH}_{2}\right)$, 
3.28 dd. (1 H, $\left.\mathrm{CH}_{2} \mathrm{I}, \mathrm{J}=10.5,5.7 \mathrm{~Hz}\right), 3.41 \mathrm{dd}$. $\left(1 \mathrm{H}, \mathrm{CH}_{2} \mathrm{I}, \mathrm{J}=10.55 .7 \mathrm{~Hz}\right), 3.85$ м. $(1 \mathrm{H}, \mathrm{CH})$, Found \%: C 42.62, $\mathrm{H}$ 6.19, I 40.93. $\mathrm{C}_{11} \mathrm{H}_{19} \mathrm{IO}_{2}$. HMDS. calculated, \%: C 42.60, H 6.17, I 40.91.

Similarly based on:

- allyl ether of cyclohexanol, propargyl alcohol and crystalline iodine was obtained $\{3-$ Iodo-2-[(2-methylbut-3-yn-2-

yl)oxy]propyl\}cyclohexane (V) by yeald of $57.6 \%$, melt.point $60-61{ }^{\circ} \mathrm{C}$ (from heptane) ). FT-IR spectra $\left(\mathrm{v} / \mathrm{sm}^{-1}\right): 560(\mathrm{C}-\mathrm{I}), 1100(\mathrm{C}-\mathrm{O}-$ $\mathrm{C}), 2100$ and $3330(\mathrm{C} \equiv \mathrm{CH}) .{ }^{1} \mathrm{H}$ NMR $(\delta$, m.d., $\left.\mathrm{CDCl}_{3}\right)$ : $1.08\left(6 \mathrm{H}, 2 \mathrm{CH}_{3}\right)$ m. $(10 \mathrm{H}$, $\left.\mathrm{C}_{6} \mathrm{H}_{10}\right), 2.45$ t. $(1 \mathrm{H}, \equiv \mathrm{CH}), 3.28$ dd. $\left(1 \mathrm{H}, \mathrm{CH}_{2} \mathrm{I}\right.$, $\mathrm{J}=10.55 .7 \mathrm{~Hz}), 3.45 \mathrm{~d}(2 \mathrm{H}, 2 \mathrm{CHO}), 3.65 \mathrm{~m}$. $(2 \mathrm{H} 2 \mathrm{CHO})$, Found \%: C 48.03, H 6.64, I
36.25, $\mathrm{C}_{14} \mathrm{H}_{23} \mathrm{IO}_{2}$. HMDS. calculated,\%: C 48.01, H 6.62, I 36.23.

- allyl ether of phenol, propargyl alcohol and crystalline iodine was obtained 3-Iodo-2 [(2-methylbut-3-yn-2-yl) oxy] propyl\} benzene (VI) by yeald of $67.2 \%$, t. boil. 115 ${ }^{\circ} \mathrm{C} \quad(2 \mathrm{mmHg}$ art. $), d_{4}^{20} 1.4502, \quad \eta_{20}^{D} 1.5450$, $\mathrm{MR}_{\mathrm{D}}$ 68.93, calculated 68.93 FT-IR spectra $\left(\mathrm{v} / \mathrm{sm}^{-1}\right): 560(\mathrm{C}-\mathrm{I}), 1100(\mathrm{C}-\mathrm{O}-\mathrm{C}), 2100$ and $3330(\mathrm{C} \equiv \mathrm{CH}), 1515,1620,3060,3080\left(\mathrm{C}_{6} \mathrm{H}_{5}\right)$. ${ }^{1} \mathrm{H}$ NMR $\left(\delta, \mathrm{ppm}, \mathrm{CDCl}_{3}\right)$ : $\left(\delta\right.$, m.d., $\left.\mathrm{CDCl}_{3}\right)$ : 2.5 t. $(1 \mathrm{H}, \equiv \mathrm{CH}, \mathrm{J}=2.4 \mathrm{~Hz}), 3.41$ d. $(2 \mathrm{H}, 2$ $\left.\mathrm{CH}_{2} \mathrm{O}\right), 3.28$ dd. $\left(1 \mathrm{H}, \mathrm{CH}_{2} \mathrm{I}, \mathrm{J}=10.55 .7 \mathrm{~Hz}\right)$, $7.15 \mathrm{~m} .\left(5 \mathrm{H}, \mathrm{C}_{6} \mathrm{H}_{5}\right)$. Found \%: C 48.87, $\mathrm{H}$ 5.02, I 36.89, $\quad \mathrm{C}_{14} \mathrm{H}_{17} \mathrm{IO}_{2}$. HMDS. calculated, \%: C 48.85, H 4.98, I 36.87.

\section{References}

1. Talybov G.M., Mekhtieva V.Z., Karaev S.F. Iodoalkoxylation of Cyclohexene with Allyl and Propargyl Alcohols. Russ. J. Org. Chem. 2001, vol. 37, no. 4, p. 600. doi.10.1023/A:1012462709589

2. Talybov G.M., Nurieva U.G., Karaev S.F. New Aryl-substituted 1,4-Dioxanes Prepared from Propargyl Ethers of (Bromomethyl)-(phenylalkyl)methanols. Russ. J. Org. Chem. 2003, vol. 39(8), pp. 1206-1208.

3. Kemp J.E.G. In Comprehensi Ve Organic Synthesis; Trost, B. M., Fleming, I.; Eds.; Pergamon: Oxford, UK, 1991; vol. 7, pp. 469-513.

4. Tatsuo KaIho. IodIne ChemIstry and applIcatIons. Published by John Wiley \& Sons, Inc., Hoboken, New Jersey. 2015, p. 661.

5. Indranirekha Saikia, Arun Jyoti Borah, and Prodeep Phukan. Use of Bromine and Bromo-Organic Compounds in Organic
Synthesis. Chem. Rev. 2016, vol. 116, pp. 6837-7042.

DOI: 10.1021/acs.chemrev.5b00400.

6. Khaire M.V., Nilavan M., Jain A. Comparative study of rust prevention performance of different rust preventive oils based on type of carriers. IOSR Journal of Mechanical and Civil Engineering, 2016, vol. 13, issue 1, pp.82-90.

7. Karaev S.F., Talybov G.M., Kulieva D.M. Antimicrobial properties of propargyloxy derivatives of organic halides. Medical Journal "VITA", 2000, no.2, pp. 40-41.

8. Karaev S.F., Talybov G.M., Teymurova R.A. Synthesis and antitumor properties of carbocyclic esters of N-substituted 4amino-2-butyn-1-tins. Azerbaijan journal of oncology and related sciences. 1995, vol. 1 , no. 1,2 , pp. 60-61.

9. Ionin B.I., Ershov B.A., Koltsov A.I. NMR spectroscopy in organic chemistry.

Leningrad: Himiya publ., 1983. 271 p.

\section{3-ORQANILOKSI-1-PROPENLORİN DIMETILASETİLENKARBINNOL MÜHİTINDӘ HALOGENALKOKSIDLOŞDIRILMOSI}

\section{A.R. Ozizbəyli, G.M. Talıbov, E.H. Mommədbəyli}

AMEA akam. Y.H. Mammadaliyev ad. Neft-Kimya Proseslari Institutu

AZ 1025, Bakl ş., Xocalı pr. 30

e-mail:ezizbeyli83@mail.ru,ahmed_adna@rambler.ru,eldar_mamedbeyli@mail.ru 
NMR spektrlarin noticalarina göra dimetilasetilenkarbinolda N-bromsuksinimid (va ya kristal yod) ila 3orqaniloksi-1-propenlorin alkoksihalogenlaşməsi ikiqat rabitə üzra regioselektiv gedir. Reaksiya aşağl temperaturda adi buzla soyudularaq aparllır. Osas reaksiya mahsullarl 3-brom(yod)-1,2-propandiol diefirlari yüksək çıxımla alınır. Müəyyən edilib ki, allil efirlarinin propinolla alkoksihalogenlaşma reaksiyası halogen atomunun $C=C$ rabitəsinə regioselektiv birlaşməsi ila həyata keçir. Bromsaxlayan birlaşməlarin məhsuldarlı̆̆ yodsaxlayan analoqlarından daha yüksəkdir. Yod tərkibli məhsullar bromlu analoqlarından daha yüksok temperaturda qaynayır. Reaksiya məhsullarında halogen atomu doymamıs spirtlo avaz olunmur. Reaksiya nazik layl xromatoqrafiya vasitasila müşahida edilir. Bu tip birlaşmalar üzvi kimyada va bioloji aktiv birlaşmalar kimi istifado olunur.

Açar sözlar: alkoksihalogenlaşma, allil efirlari, propinol, regioselektivlik, dimetilasetilenkarbinol

\section{ГАЛОГЕНАЛКОКСИЛИРОВАНИЕ 3-ОРГАНИЛОКСИ-1-ПРОПЕНОВ В СРЕДЕ ДИМЕТИЛАЦЕТИЛЕНКАРБИНОЛА}

\section{А.Р. Азизбейли, Г.М. Тальюбов, Э.Г. Мамедбейли}

Институт нефтехимических прочессов им. акад. Ю.Г. Мамедалиева

AZ 1025, г. Баку пр. Ходжальы, 30

e-mail:ezizbeyli83@mail.ru,ahmed_adna@rambler.ru,eldar_mamedbeyli@mail.ru

Алкоксигалогенирование 3-органилокси-1-пропенов N-бромсукиинимидом (или кристаллическим иодом) в среде диметилацетиленкарбинола по данными ЯМР спектров протекает региоселективно по двойной связи. Реакиию проводят при низкой температуре, охлаждая реакиионную смесь обычным льдом. Основные продукты реакиии - диэфиры-3-бром(иод)-1,2пропандиола образуются с высокими выходами. Установлено, что алкоксигалогенирование аллиловых эфиров пропинолом протекает региоселективно с фиксацией атома галогена у периферийного атома углерода связи $C=C$. Выходы бромсодержащих продуктов выме их иоданалогов. Иодсодержащие продукты кипят более высокой температуре, чем их броманалоги. B продуктах реакиии не наблюдаются вещества с заменой атома галогена непредельным спиртом. За ходом реакции следили с помощью тонкослойной хроматографии. Синтезированные соединения используются в качестве биологически активных соединений.

Ключевые слова: алкоксигалогенирование, пропинол, диэфиры 3-бром(иод)-1,2-пропандиола, диметилацетиленкарбинол 